\title{
Prioritized Broadcast Contention Control in VANET
}

\author{
Fei Ye*, Raymond Yim ${ }^{\dagger}$, Jianlin Guo ${ }^{\dagger}$, Jinyun Zhang ${ }^{\dagger}$ and Sumit Roy* \\ * University of Washington, Seattle, WA 98195. \\ $\dagger$ Mitsubishi Elect. Research Labs, Cambridge, MA 02139. \\ Emails: $\{$ fye, sroy\}@u.washington.edu, $\{y i m$, guo, jzhang\}@merl.com
}

\begin{abstract}
Reliable and timely multi-hop propagation of messages among vehicles is essential for a safer and greener transportation system. Various broadcast-based forwarding strategies are envisioned for infrastructure-less vehicle-to-vehicle (v2v) communications. This paper proposes a prioritized broadcast contention control (PBCC) module/layer that provides reliable and low latency multi-hop connection. The PBCC forwarding algorithm optimizes the back-off distribution to improve the probability of successful broadcast and prioritizes forwarders based on location information. This module can be implemented in WAVE devices with minimum system modification. We integrate simple vehicular mobility models into ns-2 and implement a WAVE/802.11p communication protocol stack. Extensive simulations demonstrate PBCC's superiority in multi-hop delay.
\end{abstract}

\section{INTRODUCTION}

Since 1994, the number of fatal vehicle crashes in the United States annually has never fallen below 35,000 [1]. Statistic shows that a large amount of road accidents are primarily caused by incorrect decision of drivers as they fail to anticipate hazardous conditions on the roads ahead. Specifically, driver's line-of-sight vision is often blocked by preceding vehicles, and bad weather conditions can further reduce visibility. Using wireless communications, cooperative collision avoidance will focuses on how in-time information may be communicated beyond single hop range. The Federal Communications Commission established licensing and service rules for the Dedicated Short Range Communications (DSRC) Service [2] in the $5.9 \mathrm{GHz}$ band in 2003. Meanwhile, the IEEE 802.11p amendment [3] is proposed to specify the PHY and MAC layers for Wireless Access in Vehicular Environments (WAVE), and the IEEE 1609 standards [4] define an architecture and a complementary standardized set of services and interfaces that collectively enable vehicular networking.

Typically, a source vehicle generates an emergency message without the knowledge of potential receivers. In the interests of source anonymity, 802.11p broadcast without acknowledge requirement is used. Upon receiving the source's broadcast, nodes will forward/rebroadcast independently, leading to occasional packet collisions. Back-off strategy is used to alleviate such inefficiencies. Furthermore, since a vehicle may possibly receive the same message from multiple rebroadcasts, redundancy suppressing can be used to improve overall utilization, which in turns improves end-to-end latency.

Though there are work that improve the MAC and forwarding algorithm for VANETs, a layer that explicitly provides reliable multi-hop $\mathrm{V} 2 \mathrm{~V}$ connection is still missing in a WAVE
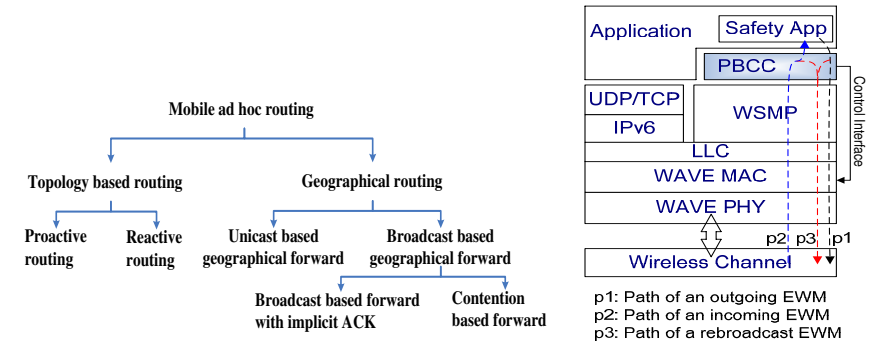

Fig. 1. Mobile ad hoc routing and $\mathrm{v} 2 \mathrm{v}$ forwarding strategies.

Fig. 2. WAVE protocol stack with the PBCC module.

communication protocol stack. In this paper, a Prioritized Broadcast Contention Control (PBCC) module/layer (Fig. 2) is proposed. This module processes both outgoing and incoming WAVE short messages, hence, handles single broadcast, periodic rebroadcast and multi-hop forwarding. These operations require neither MAC signalling nor explicit coordination among vehicles. The main contribution is three fold:

- The architecture of a PBCC module that provides reliable $\mathrm{V} 2 \mathrm{~V}$ connection. It can be implemented in WAVE devices with minimum system modification.

- A prioritized broadcast algorithm in the PBCC module that calculates the optimal distribution of back-off value for message forwarding.

- We integrate simple vehicular mobility models into network simulator ns-2 to create a reliable, open source, lightweight vehicular network simulator.

\section{RELATED WORK}

A taxonomy of mobile ad hoc routing is presented in Fig. 1. By utilizing location information, the category of geographical routing [5] is suitable for VANETs. If the sender determines a forwarder prior to unicasting to that forwarder, it is unicast based geographical forward. For example, in [6], the source identifies the furthest one hop receiver using a Request-toBroadcast and Clear-to-Broadcast mechanism. Otherwise, in broadcast based geographical forward, all immediate neighbors of the source will contend to be a forwarder in a distributed way. In broadcast based forward with implicit ACK [7], [8], a receiver periodically rebroadcasts a message until a duplicate message from any subsequent vehicle (implicit $\mathrm{ACK}$ ) is received. Solutions in this category suffer from collision and inefficient rebroadcast due to no preference among forwarding candidates. An inefficient rebroadcast refers to the case that a vehicle close to the source first accesses the channel and suppresses other candidates. Contention based forward 
is an improvement on the above. In [9], a delay inversely proportional to the distance from the source is added to relieve collision and inefficient broadcast problems. This method needs more careful consideration since introducing additional delay is counter to our ultimate goal of lower latency. Hence, a set of innovations are needed to integrate the core idea into MAC layer back-off inherent in the 802.11 DCF. As illustrated in Table I, various protocols in this category [10]-[14] identify themselves through different kernel functions in determining the contention window size $(\mathrm{CW})$ and/or the back-off value $b$, based on sender's and receiver's positions, $\operatorname{pos}_{s}, \operatorname{pos}_{r}$.

Finally, research on VANET still relies significantly on simulation. An ideal VANET simulation should closely couple packet exchanges with vehicular mobility pattern. Only recently, has some progress towards this goal been achieved; one effort (GMSF [15]) feeds network simulators with vehicle traces generated by traffic simulators. More advanced approaches include coupled simulators (TraNS [16]) and integrated ones (InVeNTSim [17]). The first category couples existing network and traffic simulators through specific interfaces to full-scale interaction between them. The latter category integrates key traffic components into network simulators to create lightweight tools. Our simulator falls in this final category - simple mobility models are integrated into ns-2.33 where a WAVE/802.11p protocol stack is also implemented.

TABLE I

THE KERNEL FUNCTIONS IN VARIOUS CONTENTION BASED FORWARD STRATEGIES

\begin{tabular}{|c|c|c|}
\hline Strategies & Kernel function $f(\star)$ & Comments \\
\hline$[10]$ & $b=T\left(1-\frac{\mid p o s_{r}-p_{0 s_{s} \mid}}{r}\right)$ & $\begin{array}{l}T \text { is the maximum forwarding delay, } \\
r \text { is the transmission range. }\end{array}$ \\
\hline [11] & $b=\min \left(\frac{a}{2}, a \exp \left(\frac{\mid p o s_{r}-\text { pos }_{s} \mid}{r}\right)\right)$ & $a$ is a protocol parameter. \\
\hline [13] & $b=\frac{r-\left|p^{2} s_{r}-\operatorname{pos}_{s}\right|}{r}\left(C W_{\max }\right.$ & \\
\hline [12] [14] & $\begin{array}{l}{\left[0, c w_{1}\right],\left[c w_{1}+1, c w_{2}\right], \ldots,} \\
{\left[c w_{m-1}+1, c w_{m}\right], \text { for } m \text { zones. }}\end{array}$ & Disjoint $\mathrm{CW}$ assignment. \\
\hline [12] & $\begin{array}{l}{\left[0, c w_{1}\right],\left[0, c w_{2}\right], \ldots,\left[0, c w_{m}\right]} \\
\text { for } m \text { zones, } c w_{1}<\cdots<c w_{m} .\end{array}$ & Overlapped (EDCA alike) $\mathrm{CW}$ assignment. \\
\hline
\end{tabular}

\section{PRIORITIZED BROADCAST CONTENTION CONTROL}

We study a VANET consisting of vehicles equipped with GPS and DSRC radios. Each on-board DSRC device has an enhanced WAVE based communication protocol stack as is illustrated in Fig. 2. All legacy unicast packets (i.e. data traffic) go through the left side as if they are in a TCP/IP stack. All WAVE messages designed for vehicular networking go through a thinner stack: from the WAVE Short Messages and Protocol layer (WSMP), to logical link control layer (LLC), and last to WAVE MAC and PHY. The WSMP layer issues short messages as single-hop broadcast in the interest of source anonymity, relying on our PBCC module for reliable singlehop and multi-hop connections. As Fig. 3 shows, the PBCC module has the following three major functions:

Initiate and periodically rebroadcast a warning message: After acquiring an emergency warning message (EWM) from upper applications, this module starts a rebroadcast process before passes it down to the WSMP layer. The rebroadcast process periodically updates and rebroadcasts this EWM, until an implicit ACK is heard or a predefined limit is reached.
Process an incoming warning message: When receive an EWM from lower layers, this module first determines whether it is an implicit ACK. If so, it aborts corresponding rebroadcast process if one exists. Otherwise, it decides if the EWM needs to be forwarded before sends it up. If so, a rebroadcast process is started.

Prioritized broadcast contention control: A rebroadcast packet assembled at the PBCC module may be either a locally generated message or a forwarding message. This message may be directly sent down or prioritized. The prioritization process (via the prioritized broadcast contention control algorithm) usually applies to forwarding messages.

Zone partition: In the forwarding algorithm, the expected communication range $r$ is partitioned into $m$ equal zones $z_{1}, z_{2}, \ldots, z_{m}$, where $z_{1}$ is the closest zone. Zone index is computed using $i=\left\lceil\frac{d}{r} m\right\rceil$, where $d$ is the distance to the source, and $\lceil\cdot 7$ is the ceiling function. In this way, each vehicle independently estimates its zone index upon reception of an EWM.

Optimal back-off strategy: Vehicles are prioritized based on their zone index through back-off. Assume $n$ vehicles are uniformly distributed in $m$ zones and there are $s(s>m)$ available back-off slots. Let $P_{i, j}$ denote the probability that a vehicle in zone $z_{i}$ chooses back-off value ${ }^{1} j$. We want to find the optimal probability matrix $P_{m \times s}$, which maximizes the probability of successful broadcast, and statistically ensure higher priority for vehicles in further zones. The probability matrix filling algorithm introduced in [18] is summarize in Algorithm 1, and its optimality is summarized in Theorem 1.

Theorem 1: If $n$ uniformly distributed vehicles independently select their back-off values from $\{1, \ldots, s\}$, and any two or more transmissions in the same slot cause a collision, a vehicle's probability of successful broadcast is

$$
P_{s}=\sum_{j=1}^{s} q_{j}\left(1-q_{j}\right)^{n-1} \leq\left(1-\frac{1}{s}\right)^{n-1},
$$

where $q_{j}=\frac{1}{m} \sum_{i=1}^{m} P_{i, j}$, and the equality is achieved if and only if $q_{1}=q_{2}=\cdots=q_{s}=1 / \mathrm{s}$.

Any probability matrix generated by Algorithm 1 achieves the equality, and also statistically ensures early channel access for vehicles that are further away, that is:

$$
\begin{aligned}
& \sum_{j=0}^{k} P_{a, j} \geq \sum_{j=0}^{k} P_{b, j}, \\
& \forall k \in\{1,2, \ldots, s\}, \forall a>b, a, b \in\{1,2, \ldots, m\} .
\end{aligned}
$$

Some existing forwarding algorithms are special cases of Algorithm 1. Broadcast Forward with Implicit ACK [7], [8] is the degenerate 1-zone case $\left(P_{1, j}=\frac{1}{s}, \forall j \in\{1,2, \ldots, s\}\right)$, where the back-off value is uniform in $[0, s-1]$. In geobased Time Division Multiple Access (TDMA) solution [17], a unique slot is assigned to vehicles within a small geographical zone. With sufficient large number of zones, the PBCC algorithm approximates such geo-based TDMA solution.

\footnotetext{
${ }^{1}$ Back-off value usually starts from 0 . For simplicity, we assume back-off value $b \in\{1,2, \ldots, s\}$ in the analysis.
} 


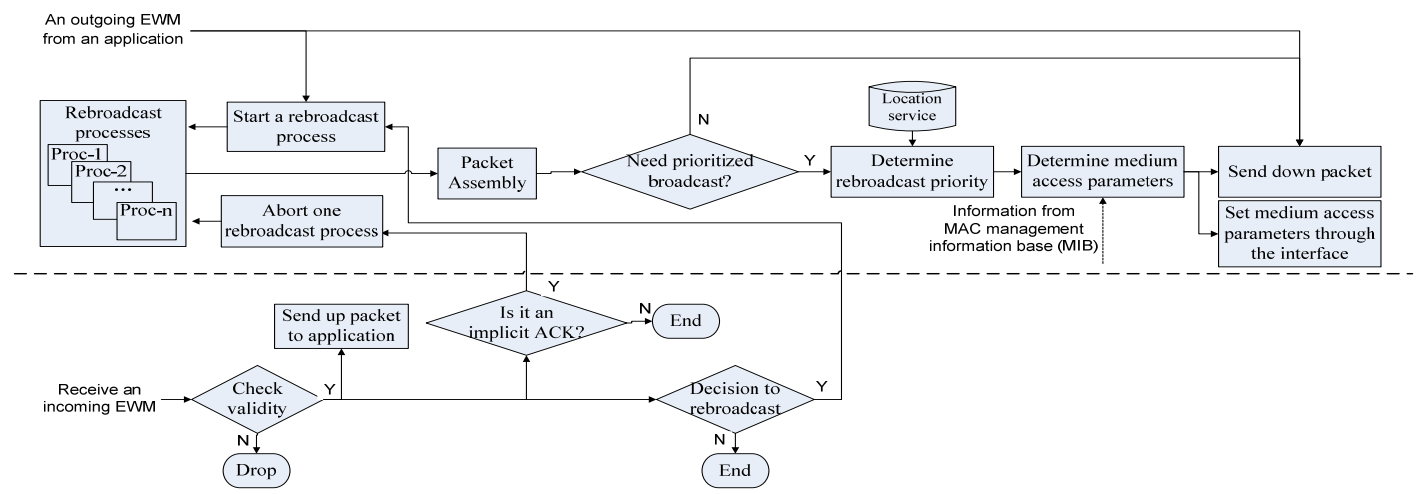

Fig. 3. Processing of outgoing and incoming WAVE short messages in the PBCC module.

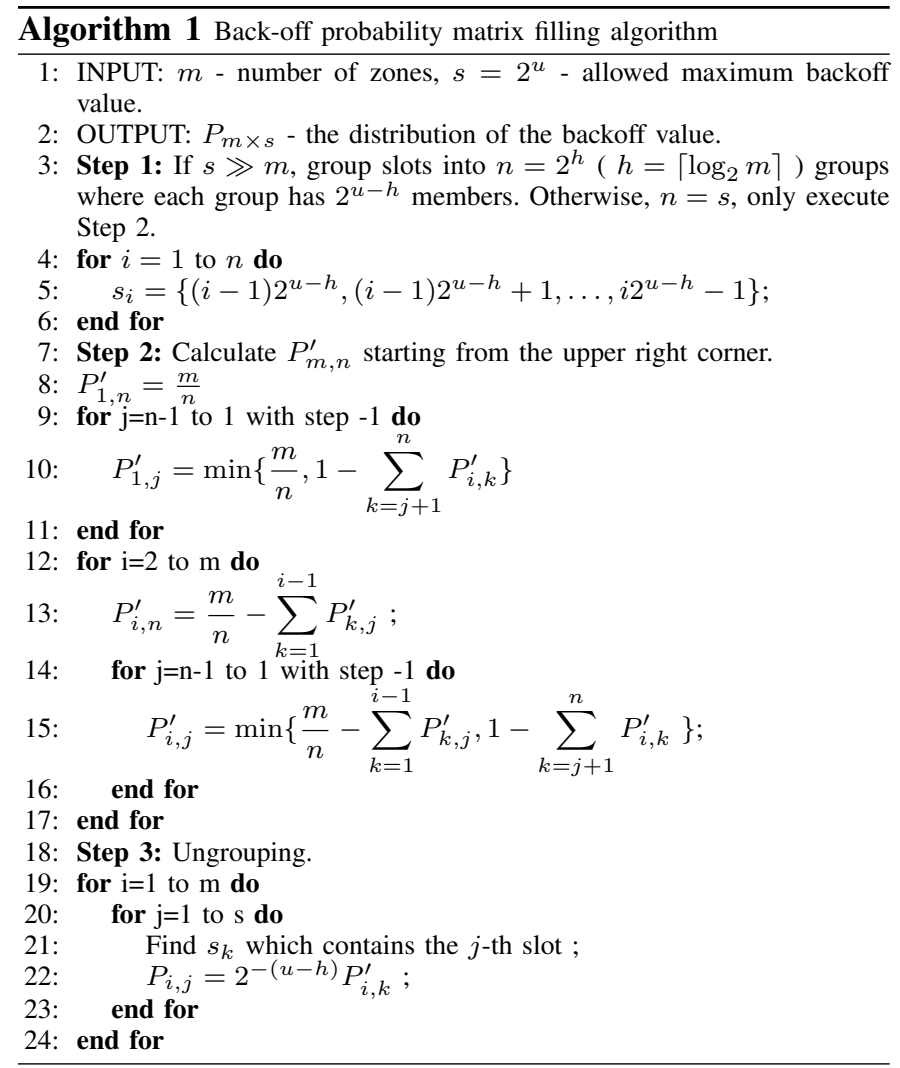

\section{Simulation}

\section{A. Integrated Simulation Platform}

Key vehicular mobility components are integrated into ns2.33 to create a lightweight tool for network centric vehicular network research. On the vehicle mobility side, each vehicle follows its preceding vehicle at a constant speed, and has a vision range. A vehicle may be notified in 3 ways, either sees any brake light, or receives a valid warning message, or identifies that the inter-vehicle spacing is too small. Once notified, appropriate reactions (i.e. adaptive deceleration) are preformed after a certain reaction time. The actual deceleration is capped by the maximum deceleration rate. The state transition diagram is illustrated in Fig. 4. Though the simulator can take other sophisticated car following models as plug-ins, the gain to our specific scenario is very marginal. As we will see in the next subsection, the message propagation event that we are interested in usually ends in less than one second. Hence, the location difference predicted by different mobility models is rather small in such a short duration.

On the communication side, the underlying PHY layer monitors the accumulated interference level and adopts a capture reception model. A control interface is provided at the MAC layer, which allows the transmission parameters, such as power, AIFSN, CW, and back-off value to be configured at a per packet basis. Other MAC and PHY parameters are set according to the $802.11 \mathrm{p}$ standard (Table II). A WSMP layer is implemented in parallel with TCP/IP stack. The proposed PBCC module is stacked on top of the WSMP layer. This module has three interfaces: two data interfaces to upper and lower layers for outgoing and incoming messages, and one control interface connected to the MAC. This control interface is the only modification required when add this module into real WAVE/DSRC systems.

\section{B. Performance Evaluation}

We simulation vehicles on a strip-shaped 3-lane freeway. Since collision depth heavily relies on drivers' behavior that is difficult to model, we show multi-hop warning message propagation delay. The plot of delay versus vehicle index explicitly gives the notified range under any delay restriction. In most figures, $90 \%$ confidence interval is shown. The following three message forwarding protocols are compared: (1) PBCC algorithm, (2) BF-ACK, a broadcast based forward strategy with implicit ACK, and (3) CBF-CW, a general contention based forward strategy. In CBF-CW, the transmission range is also partitioned into $m$ zones, and vehicles in zone $z_{1}, z_{2}, \ldots, z_{m}$ are assigned different contention window sizes $\left[0, c w_{m}\right],\left[0, c w_{m-1}\right], \ldots,\left[0, c w_{1}\right], c w_{1}<\cdots<c w_{m}$. For more details about BF-ACK and CBF-CW, please refer to [7], [8] and [12].

The impact of background traffic (BGT): It has been shown in [8] that legacy IEEE 802.11 DCF is not suitable for $v 2 \mathrm{v}$ communication due to no prioritization. Hence, in the following, all algorithms implicitly leverage on the EDCA function in the WAVE MAC, i.e. BGT are forced to use larger AIFSN, $\mathrm{CW}_{\min }$ and $\mathrm{CW}_{\max }$. In simulation, each vehicle is exchanging $20 \mathrm{kbps}$ or $60 \mathrm{kbps}$ BGT with one of its neighbors. In BF$\mathrm{ACK}$, the back-off value is uniform in $[0,63]$, while in 3-zone 


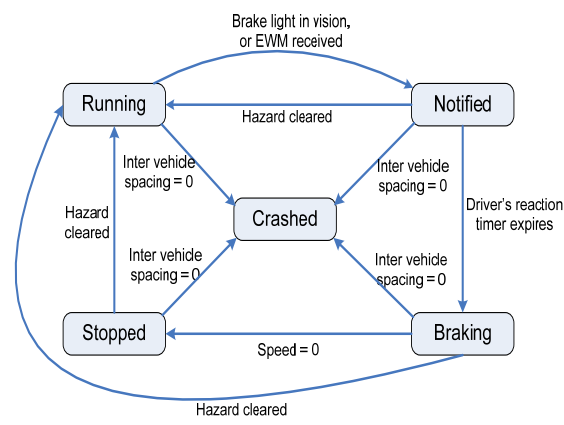

Fig. 4. A vehicle's state transition diagram in the integrated simulator.

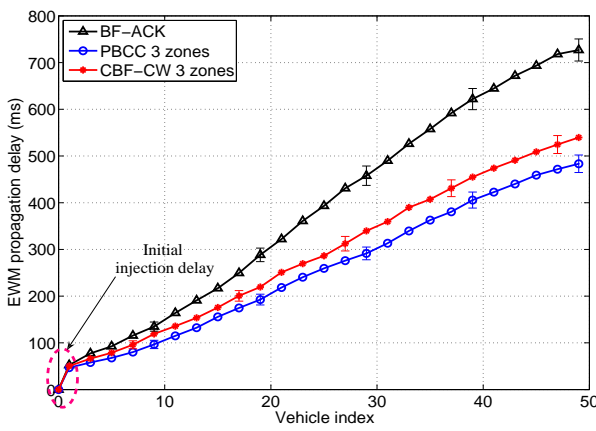

Fig. 7. Performance comparison in presence of heart beats in a dense scenario.

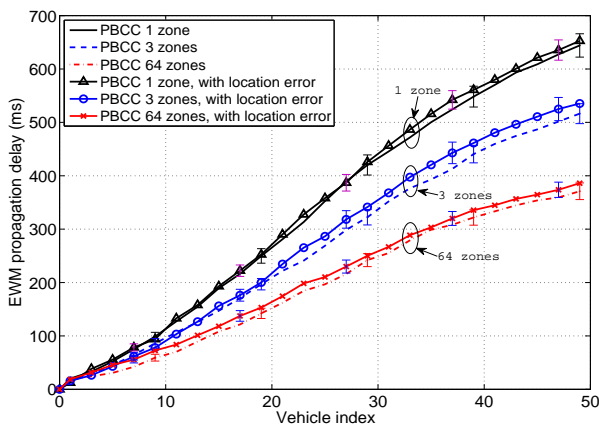

Fig. 10. The impact of $N(0,10)$ location error in a sparse scenario.

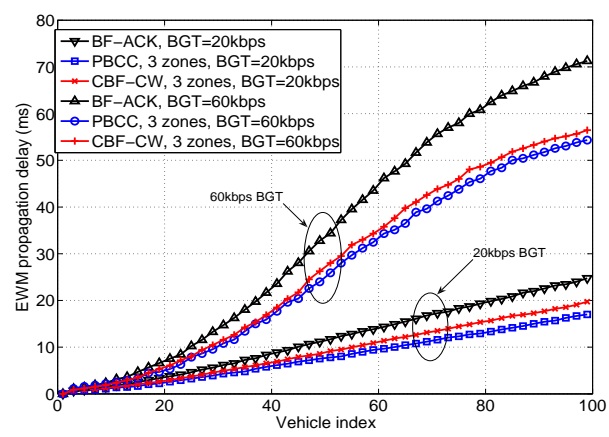

Fig. 5. Performance comparison in presence of $20 \mathrm{kbps}$ and $60 \mathrm{kbps}$ background traffic.

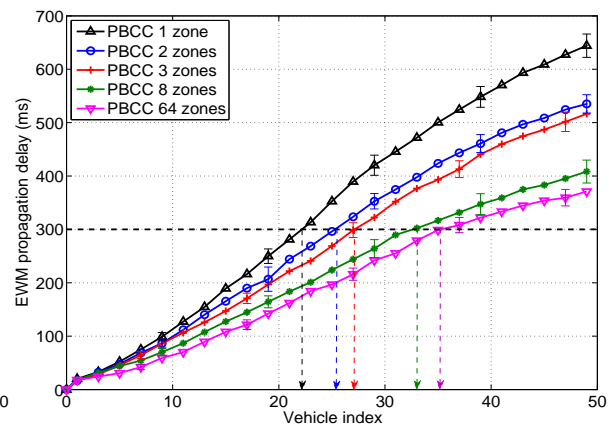

Fig. 8. The impact of zone numbers under 20pps heart beats in a sparse scenario.

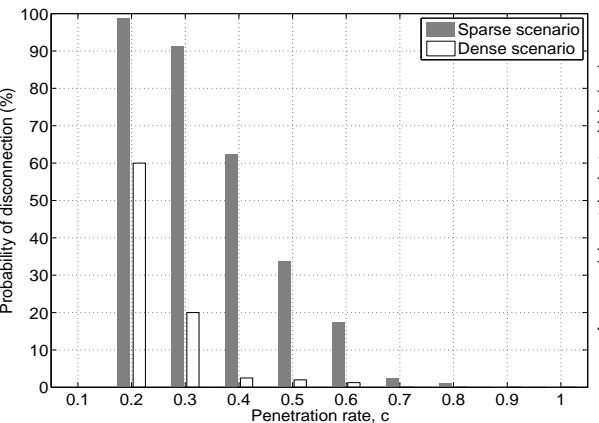

Fig. 11. Probability of disconnection vs. market penetration rate in sparse and dense scenarios.

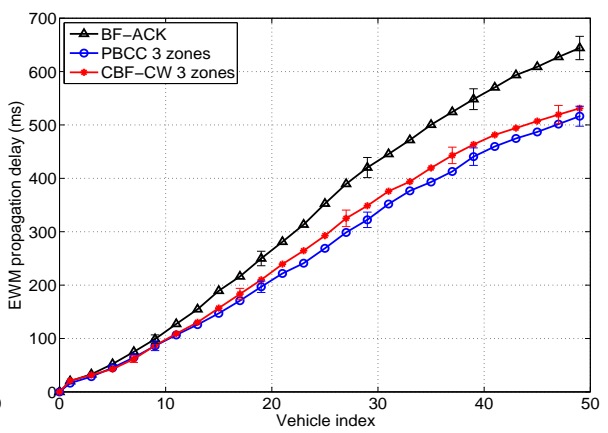

Fig. 6. Performance comparison in presence of heart beats in a sparse scenario.

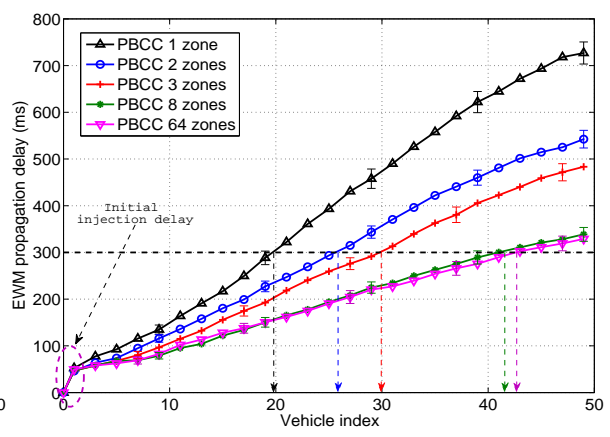

Fig. 9. The impact of zone numbers under 20pps heart beats in a dense scenario.

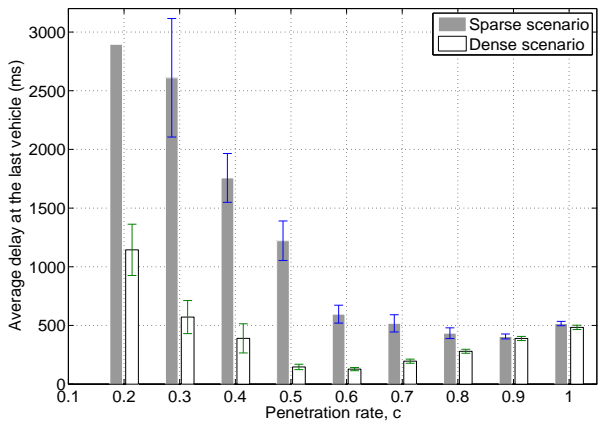

Fig. 12. Propagation delay vs. market penetration rate in sparse and dense scenarios.
CBF-CW, the contention window assignment is $[0,63],[0,42]$, and $[0,31]$. As expected, regardless of the amount of BGT, prioritizing vehicles leads to lower delay in both $\mathrm{PBCC}$ and CBF-CW in Fig. 5. A closer look reveals that BGT is well handled by EDCA in the WAVE MAC, and the delay (under $100 \mathrm{~ms}$ at the 100th vehicle) is small enough for most vehicular applications.

The impact of heart beat messages: Vehicles are required to periodically exchange cooperative awareness messages (CAM), also known as heart beats, which contain position, motion and control information. Heart beats may significantly degrade system performance, since they are close to the same priority level as the EWM. In the simulation, each vehicle is broadcasting heart beats at 20 packet-per-second (pps). Vehicle density is varied ${ }^{2}$ in a 3-lane scenario instead of changing each vehicle's packet generating rate as before. Connecting Fig. 6 and Fig. 7 with Fig. 5, we see that the

\footnotetext{
${ }^{2}$ In a sparse scenario, the 50th vehicle corresponds to $2250 \mathrm{~m}$ in distance approximately, while $1250 \mathrm{~m}$ in a dense scenario.
}

accumulated delay in presence of heart beats is an order of magnitude larger than that with only BGT. Hence, regulation of the amount of heart beat messages on a WAVE channel is definitely needed. Moreover, there is an initial injection delay for EWMs. When inject a new and unexpected warning message, a single source could not prioritize it over heart beats from neighboring vehicles. Hence, this injection delay depends on density, and is relieved by neither rebroadcasting nor forwarding. In Fig. 7, one could see that this delay remains the same for all three algorithms. Further simulations show that injection delay closely relates to vehicle density, heart beat frequency, and rebroadcast interval.

The impact of zone numbers: An immediate relevant thinking is increasing zone numbers to combat the performance degradation. In Fig. 8 and Fig. 9, various zone numbers, hence different zone granularities, are evaluated in sparse and dense scenarios in presence of heart beats. From no prioritization (1-zone case) to 2-zone case, we observe significant delay decrease and alert range extension in all cases. However, 
further increasing zone numbers incurs diminishing marginal gain. This is because (1) contention and interference from heart beats, and (2) prioritization through back-off is in statistical sense, and its accuracy decreases as more prioritization levels (zones) are demanded from a limited number of back-off slots. Since more zones requires more accurate location information, GPS accuracy is another limitation on having too many zones. These trade-offs should be carefully studied when determining zone numbers for different applications in various scenarios. For instance, to inform 30 neighbors (which approximately spans $1350 \mathrm{~m}$ in a sparse and $750 \mathrm{~m}$ in a dense scenario) within $300 \mathrm{~ms}$ delay on average, 8 zones are required in a sparse scenario, while 3 zones are enough in a dense case.

Practical issues: Systems that rely on location information are often susceptible to location errors. The forwarding algorithm in the PBCC module is fully distributed, and requires a successfully rebroadcast to suppress other potential forwarders at each hop. If the optimal forwarder misses its chance due to location error, one of sub-optimal forwarders will take act, lending needed robustness to algorithm operation. Fig. 10 shows the robustness in presence of a Gaussian location error $N(0,10)$. Besides, market penetration rate $c$, defined as the percentage of vehicles which are equipped with DSRC radios, is a key factor in determining multi-hop connectivity ${ }^{3}$, mainly for data services. As shown in Fig. 11, the probability of disconnection becomes significant when penetration rate drops below a certain threshold that is vehicle density dependent. Ironically, as Fig .12 shows, the delay of successful connections could be smaller when the system can tolerate a certain amount of connection failures.

\section{CONCLUSION}

This paper proposes a prioritized broadcast contention control (PBCC) module for reliable and low latency $\mathrm{v} 2 \mathrm{v}$ connection. During multi-hop forward, the prioritized forwarding algorithm running in the PBCC module at each vehicle independently calculates the host's optimal back-off distribution to prioritize potential forwarders that are further away from the source. Most importantly, this module can be implemented as an independent layer in a WAVE/DSRC device. The only modification required in existing system is a control interface to the WAVE MAC layer for back-off timer manipulation. There is no need for additional signaling overhead or explicit coordination among vehicles.

To simulate a VANET, we integrate simple vehicular mobility components into ns- 2.33 to create an integrated simulator that is suitable for most network centric VANET research. Extensive simulations demonstrated the PBCC forwarding algorithm's superiority in multi-hop delay in all cases, and also revealed the following: (1) low priority background traffic is well handled by WAVE MAC, while periodical heart beats are not; (2) the trade-offs of utilizing multiple zones in the PBCC

\footnotetext{
${ }^{3} \mathrm{~A}$ disconnection is said to happen if the message fails to reach the las vehicle at simulation end (5s). Vehicles do not change their mobility pattern upon reception of a message in penetration rate simulation.
}

forwarding algorithm; (3) the PBCC forwarding algorithm has the needed robustness against location errors.

One possible extension of this work is to conduct simulations subject to realistic urban constraints, such as city map, traffic rules, $\mathrm{RF}$ attenuation due to obstacles, such as buildings.

TABLE II

SIMULATION PARAMETERS

\begin{tabular}{l|l||l|l}
\hline Road topology & Linear, 3-lane & Vehicle velocity & $25 \mathrm{~m} / \mathrm{s}$ \\
Spacing (sparse) & U[35,55]m & Spacing (dense) & U[15, 35]m \\
Maximum deceleration & U[6, 10]m/s/s & Driver's reaction time & U[1.2, 1.8]s \\
Central frequency & $5.890 \mathrm{Ghz}$ & Channel bandwidth & $10 \mathrm{Mhz}$ \\
Propagation model & Two-ray ground & Modulation scheme & BPSK \\
Capture threshold & $5 \mathrm{~dB}$ & Data packet size & 512 Bytes \\
CW min for data packet & 127 & CW max for data packet & 1023 \\
EWM size & 128 Bytes & Heart beat msg size & 256 Bytes \\
CW for EWM & {$[0,63]$} & AIFSN for EMW & 2 \\
AIFSN for data packet & 9 & EWM broadcast interval & $25 \mathrm{~ms}$ \\
BGT tx power & $100 \mathrm{~mW}$ & EWM tx power & $300 \mathrm{~mW}$ \\
\hline
\end{tabular}

\section{REFERENCES}

[1] "Fata Analysis Reporting System," http://www-fars.nhtsa.dot.gov.

[2] "Dedicated Short Range Communications (DSRC) Service in Federal Communications Commission (FCC)," http://wireless.fcc.gov/services.

[3] "IEEE P802.11p/D5.0, IEEE 802.11 Amendment 7: Wireless Access in Vehicular Environments," Nov. 2008.

[4] "IEEE Std 1609 family, IEEE Trial-Use Standard for Wireless Access in Vehicular Environments(WAVE)," Nov. 2006.

[5] M. Mauve, A. Widmer, and H. Hartenstein, "A survey on position-based routing in mobile ad hoc networks," IEEE Network, vol. 15, no. 6, pp. 30-39, 2001.

[6] G. Korkmaz, E. Ekici, and F. Ozguner, "Black-Burst-Based Multihop Broadcast Protocols for Vehicular Networks," IEEE Trans. on Veh. Tech., vol. 56, no. 5 Part 2, pp. 3159-3167, 2007.

[7] S. Biswas, R. Tatchikou, and F. Dion, "Vehicle-to-vehicle wireless communication protocols for enhancing highway traffic safety," IEEE Commun. Mag., vol. 44, no. 1, pp. 74-82, 2006.

[8] F. Ye, M. Adams, and S. Roy, "V2V wireless communication protocol for rear-end collision avoidance on highways," in Proc. of Vehi-Mobi '08 co-located with IEEE ICC, 2008.

[9] H. Füßler, J. Widmer, M. Käsemann, M. Mauve, and H. Hartenstein, "Contention-based forwarding for mobile ad hoc networks," Ad Hoc Networks, vol. 1, no. 4, pp. 351-369, 2003.

[10] H. Füßler, H. Hartenstein, J. Widmer, M. Mauve, and W. Effelsberg, "Contention-based forwarding for street scenarios," in Proc. of 1st Int'l Workshop on Intelligent Transportation, 2004.

[11] M. Nekovee, B. Bogason, and B. Res, "Reliable and effcient information dissemination in intermittently connected vehicular adhoc networks," in Proc. of IEEE VTC, 2007.

[12] C. Chiasserini, E. Fasolo, R. Furiato, R. Gaeta, M. Garetto, M. Gribadou, M. Sereno, and A. Zanella, "Smart broadcast of warning messages in vehicular ad hoc networks," in Proc. of Workshop Interno Progetto NEWCOM, 2005

[13] C. Palazzi, S. Ferretti, M. Roccetti, G. Pau, and M. Gerla, "How do you quickly choreograph inter-vehicular communications? A fast vehicle-tovehicle multi-hop broadcast algorithm, explained," in Proc. of IEEE 4th CCNC, 2007

[14] M. Zorzi and R. Rao, "Geographic random forwarding (GeRaF) for ad hoc and sensor networks: multihop performance," IEEE Trans. on Mobile Computing, vol. 2, no. 4, pp. 337-348, 2003.

[15] R. Baumann, F. Legendre, and P. Sommer, "Generic mobility simulation framework (GMSF)," in Proc. of the 1st MobilityModels, May 2008.

[16] M. Piorkowski, M. Raya, A. Lugo, P. Papadimitratos, M. Grossglauser, and J. Hubaux, "TraNS: realistic joint traffic and network simulator for VANETs," ACM SIGMOBILE Mob. Comput. and Commun. Rev., vol. 12, pp. 31-33, 2008.

[17] F. Yu and S. Biswas, "Self-configuring TDMA protocols for enhancing vehicle safety with DSRC based vehicle-to-vehicle communications," IEEE J. Sel. Area Comm., vol. 25, no. 8, pp. 1526-1537, 2007.

[18] R. Yim, J. Guo, P. Orlik, and J. Zhang, "Received Power-Based Prioritized Rebroadcasting for V2V Safety Message Dissemination," in Proc. of Int. Transport. Sys. World Congr., Sep. 2009. 